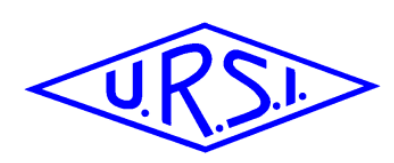

\title{
Localization of Partial Discharge by Using Received Signal Strength
}

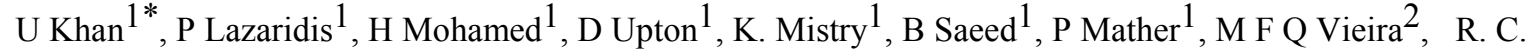 \\ Atkinson ${ }^{3}$, C. Tachtatzis ${ }^{3}$, and I A Glover ${ }^{1}$ \\ ${ }^{1}$ Department of Engineering \& Technology, University of Huddersfield, Huddersfield HD1 3DH, UK \\ ${ }^{2}$ Department of Electrical Engineering, Universidade Federal de Campina Grande, Campina Grande, Brazil \\ ${ }^{3}$ Department of Electronic and Electrical Engineering, University of Strathclyde, Glasgow G1 1XW, UK \\ E-mail: umar.khan@hud.ac.uk
}

\begin{abstract}
The term partial discharge (PD) refers to a partial breakdown in an insulator which bridges two conductors. PD occurs due to insulation defects which arise as a result of high voltage stresses or insulation cracks. Continuous monitoring of PD activity can have a significant impact towards mitigating catastrophic failures. In recent times, it has become possible to detect and locate a PD activity on an automated basis using wireless sensor technology. In this paper, a novel technique for PD detection and localization using a wireless sensor network (WSN) is presented. The localization algorithm is based on received signal strength (RSS).
\end{abstract}

Keywords - Localization; Partial discharge; RSS; UHF; $V H F ; W S N$

\section{Introduction}

PD activity takes place mainly in power transmission lines, power transformers, generators and power cables. PD arises in places such as voids, joints, cavities or delamination zones in high voltage component insulation systems [1]. The repetitive occurrence of PD activity can lead to system degradation and can affect the performance of the system and consequently may lead to the breakdown of the whole insulation system [1]. PD pulse duration is quite short and lasts typically less than $1 \mu s$ [2]. Different PD types such as corona and surface discharges are observed from electrode edges, point edges or cylindrical wires. Usually, the surface PD is observed at insulation boundaries. A common perception regarding PD activity is that it often appears in cavities or air inclusions. If a breakdown occurs due to PD, it will be preceded by consecutive PD pulses.

In recent times, it has become possible to detect and locate a PD activity on an automated basis by using the time difference of arrival (TDOA) mechanism [3] [4]. The work in [5] shows the potential use of SDR USRP N200 to locate the PD source by using the received signal strength. Despite the noisy environment, the localization results were encouraging. On the other hand, although
TDOA is effective and works correctly at the commercial level [4], the approach proposed in this paper has several advantages over this approach. Recently, the work in [6] has shown the potential use of low-cost RTL-SDR software defined radio for wideband spectrum sensing which is based on the received signal strength. The key limitation for the potential use of RTL-SDR is accuracy and sampling speed. The work in [7] presents a probability-based algorithm, and in [8] an integrated low cost detector is proposed. Free space radiometric calibration proposed in [9] is based on four different types of emulators radiating signals in the VHF-UHF range. Although a range of early warning detection systems such as [10] exist, they cannot be used for continuous monitoring. Antenna based localization proposed in [11] and [12] requires time synchronization in both cases. This is a major challenge in such schemes, especially when there are scalability requirements.

The main advantages of the proposed technology include:

- No requirement for time synchronization of receiving nodes

- Intensity-based approach that is cost effective

- Simpler approach compared to its counterparts

- Scalable approach

Keeping in view these factors, the intensity based measurement of PD seems to be an integral part of future smart grid monitoring.

\section{Algorithm Description}

The localization algorithm for PD source position estimation proposed here is based on received signal strength intensity. This method is based on the path loss model equation given below:

$R_{M}=R_{S}-10 n \log \left(\frac{d i}{d 0}\right)$

Here, $R_{M}$ is the measured signal strength by the receiving node, $R_{S}$ is the transmitted power of the source which is unknown, $n$ is the path loss index (again this is unknown, 
however it can be constrained). $d_{i}$ and $d_{0}$ are the $i t h$ and reference node distances respectively. Under an anonymous environment, the source transmitted power and the path loss exponent are both unknown and hence it is impossible to solve the above equation. To solve the problem, the ratio approach is used that eliminates the source power. The path loss exponent is still unknown but it is constrained to minimum and maximum limits. An optimization of the path loss exponent is required, however. The flowchart in Figure 1 describes the algorithm.

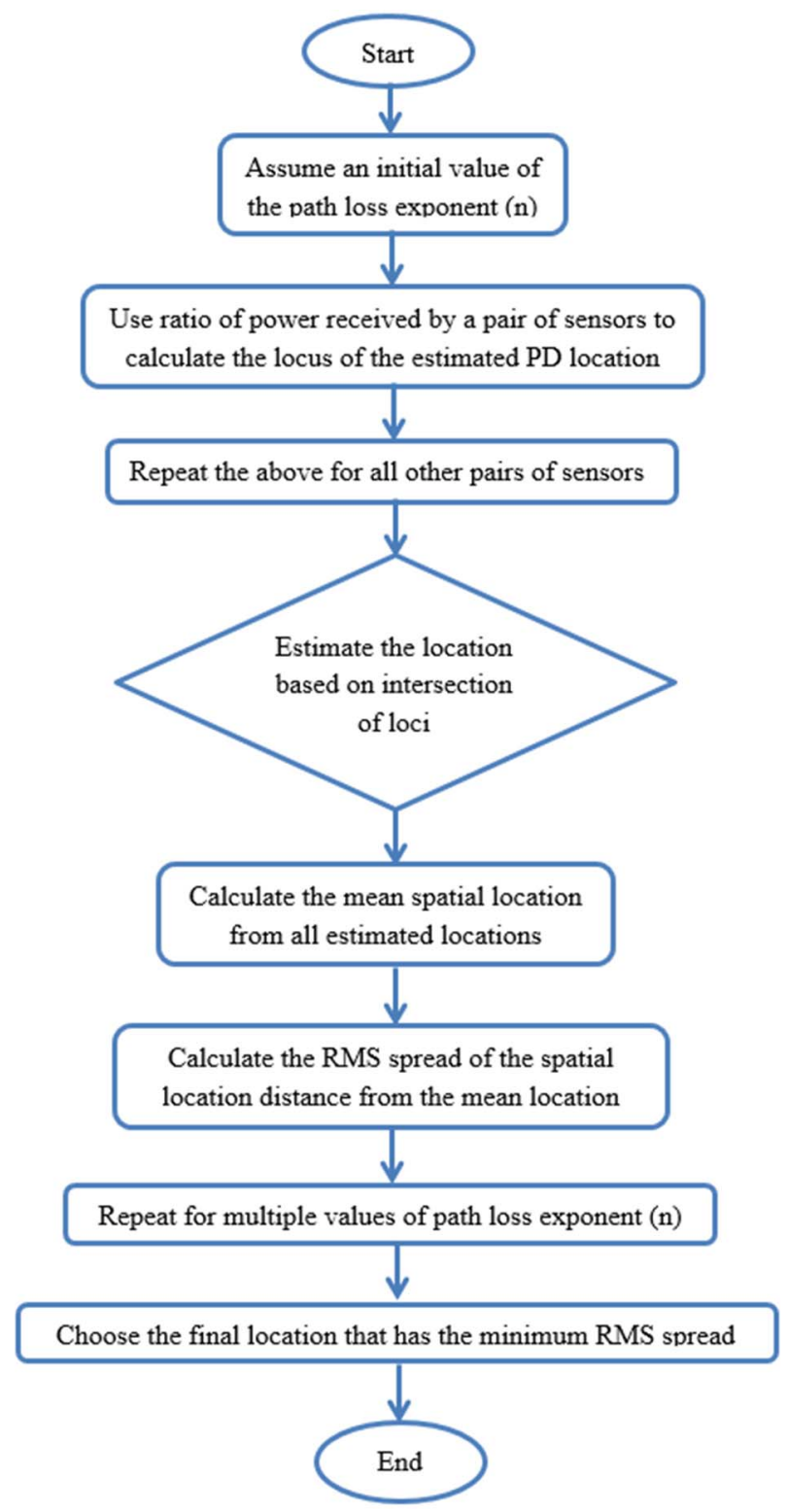

Figure 1. Location algorithm flowchart.

To optimize the path loss exponent, an initial value of the path loss exponent is chosen, e.g. 2 (free space). To calculate the locus of the estimated location, the ratio of power between a node pair is used and is repeated for all other nodes within the system. This will give multiple estimated locations. By using all these estimate locations, a mean spatial location is calculated representing the $(x, y)$ coordinates of the source. The next part is to calculate the RMS spread of spatial location distance from the mean location as given in the formula below:

$$
d_{R M S}=\sqrt{\frac{1}{N} \sum_{n=1}^{N} d_{n}^{2}}
$$

Where, $d_{n}$ is the spatial location distance from the mean estimated location and $d_{R M S}$ is the RMS spread of the spatial location distance. The process is repeated for multiple values of path loss exponent (n) by taking a reasonable step size keeping in view the computation time, e.g. 0.01. The location that will have the minimum value of RMS spread will be the estimated location of the source, and the value of the path loss exponent will be the optimized value. The mathematical modelling of the algorithm is described, given the value of $R_{M}$ in $\mathrm{dBm}$. The distance ( $d i)$ between the ith sensor node and the source can be estimated as below:

$d i=d_{0}\left[\operatorname{antilog}\left(\frac{R_{S}-R_{M}}{10 n}\right)\right]$

This problem is solved by converting $R_{S}$ into a ratio, as in [13] [14]. Let

$\operatorname{antilog}\left(R_{M} / 10\right)=P_{I}$, and

$\operatorname{antilog}\left(R_{S} / 10\right)=P_{0}$

Equation (3) can, therefore, be solved as:

$\frac{d_{1}^{2}}{d_{i}^{2}}=\frac{P_{i}^{2}}{P_{1}^{2}}$

$\frac{d_{i}}{d_{1}}=\left(\frac{P_{i}}{P_{1}}\right)^{\frac{1}{n}}$

which is further written in equation (8) as:

$\frac{d_{1}}{d_{i}}=\frac{P_{i}{ }^{\frac{1}{n}}}{P_{1} \frac{1}{n}}$

By re-arranging equation (8) for $d_{i}$, the overall expression is given in equation (9):

$d_{i}=\frac{d_{1} p_{1}^{\frac{1}{n}}}{p_{i} \frac{1}{n}}$

The intersection of the circles can be used to estimate the position of the source, which is given by equation (10):

$\left(x_{i}-x\right)^{2}+\left(y_{i}-y\right)^{2}=\frac{d_{1}^{2} p_{1}^{\frac{2}{n}}}{p_{i}^{\frac{2}{n}}}$

By using all estimated locations, the mean spatial location is calculated, and then the RMS spread of the spatial location distance from the mean location is calculated by 
using equation (2) above. For the entire range of path loss exponents, the whole process is repeated, and the value of path loss exponent $\mathrm{n}$ that provides the minimum RMS spread is used to calculate the final estimated location.

\section{The Localization Results}

The location estimation was performed via simulations in MATLAB. The algorithm requires a minimum of four sensor nodes. Simulations were performed including noise in a $20 \mathrm{~m} \times 20 \mathrm{~m}$ grid. The noise modelling was performed by using specified values of the noise figure and RF reception bandwidth. Simulations were performed for a source power of $-30 \mathrm{dBm}$ and noise figure values of $2 \mathrm{~dB}$ and $3 \mathrm{~dB}$ and bandwidths of $10 \mathrm{MHz}$ and $100 \mathrm{MHz}$, respectively. The noisy signals were created by adding to the received signal random values generated by a Gaussian distribution with zero mean and the variance calculated as:

$N=K T_{E Q} B$

Where $T_{E Q}$ is the equivalent temperature in ${ }^{\circ} \mathrm{K}$, and $\mathrm{B}$ is the bandwidth of the system in $\mathrm{Hz}$. The numbers of sensors used are initially four. A simulation with an RF bandwidth of $10 \mathrm{MHz}$ with a noise figure of $2 \mathrm{~dB}$ was carried out, and the results are shown in Figure 2. This Figure shows that there is an estimation error of around 1.19 meters.

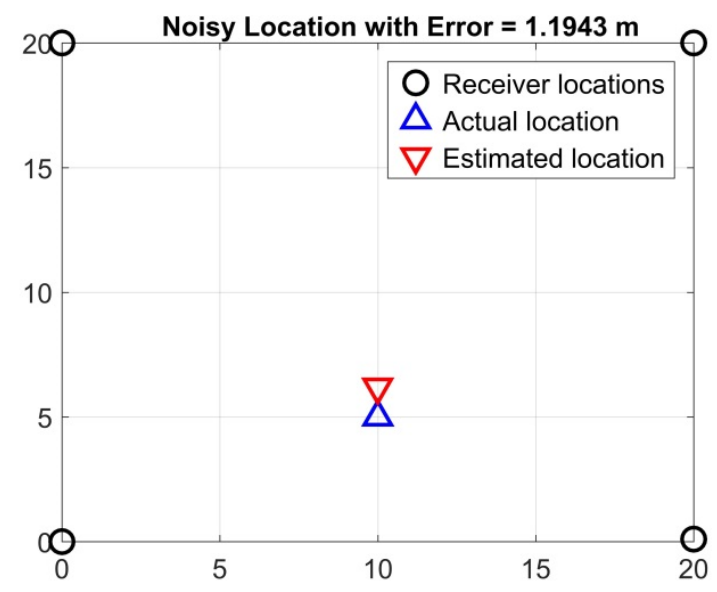

Figure 2. Simulation with Noise Figure $=2 \mathrm{~dB}$ and RF bandwidth of $10 \mathrm{MHz}$.

Figure 3 shows the results with a noise figure of $3 \mathrm{~dB}$ and an RF bandwidth of $100 \mathrm{MHz}$. This Figure shows that there is a much larger estimation error of around 2.25 meters, as expected. These results demonstrate the required specifications for the RF front-end of the system. Regarding noise calculations, random noise signals with zero mean and a fixed value of standard deviation were generated. Dealing with random numbers was considered by running the program 100 times and taking the mean of the 100 estimated values. This means that there were 100 independent runs of the program and the final location was the mean 100 independent locations.

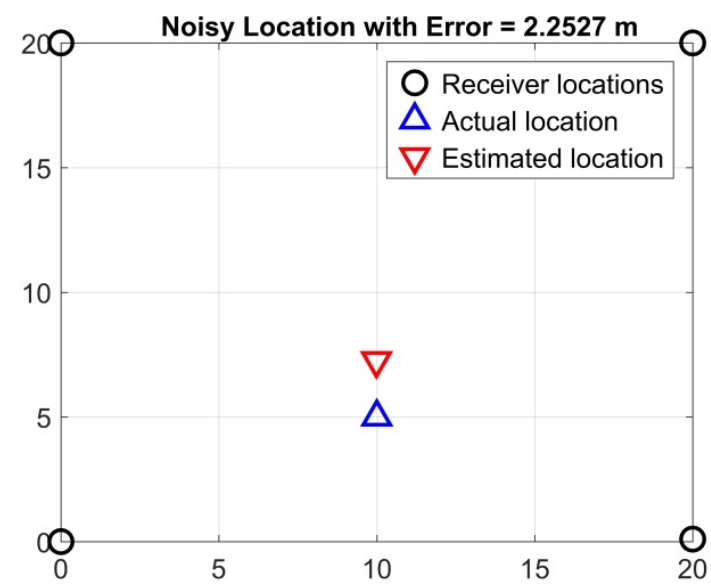

Figure 3. Simulation with Noise Figure $=3 \mathrm{~dB}$ and $\mathrm{RF}$ bandwidth of $100 \mathrm{MHz}$.

\section{Conclusion}

A novel RSS based PD localization algorithm has been proposed in this paper. Under an anonymous environment, the algorithm shows satisfactory results based on simulations. The performance of the algorithm was tested with typical values of system noise figure and RF bandwidth.

\section{Acknowledgements}

The authors acknowledge the Engineering and Physical Sciences Research Council for their support of this work under grant EP/J015873/1.

\section{References}

[1] H. Illias, S. T. Yuan, A. H. Abu Bakar, H. Mokhlis, G. Chen and P. L. Lewin, "Partial discharge patterns in high voltage insulation," in IEEE International Conference on Power and Energy (PECon), Kota Kinabalu Sabah, Malaysia, 2012.

[2] Y. Zhang, D. Upton, A. Jaber, H. Ahmed, B. Saeed, P. Mather, P. Lazaridis, A. Mopty, C. Tachtatzis, R. Atkinson, M. Judd, M. F. Q. Vieira and I. Glover, "Radiometric Wireless Sensor Network Monitoring of Partial Discharge Sources in Electrical Substations", International Journal of Distributed Sensor Networks, 11, 9, pp. 1-9, January 2015, doi: $10.1155 / 2015 / 438302$.

[3] P. J. Moore, I. E. Portugues and I. A. Glover, "Radiometric location of partial discharge sources on energized high-Voltage plant," Power Delivery, IEEE Transactions, vol. 20, no. 3, pp. 1-9, 2005. 
[4] P. Miao, X. Li, H. Hou, G. Sheng, Y. Hu and X. Jiang, "Location algorithm for partial discharge based on radio frequency (RF) antenna array," Power and Energy Engineering Conference (APPEEC), 2012 Asia-Pacific, Shanghai, China, 2012.

[5] H. Mohammed, P. Lazaridis, D. Upton, U. Khan, K. Mistry, B. Saeed, P. Mather, M. F. Q. Vieira, K. W. Barlee, D. S. W. Atkinson and I. A. Glover, "Partial Discharge Localization Based on Received Signal Strength", $23^{\text {rd }}$ International Conference on Automation and Computing (ICAC), pp. 1-4, Huddersfield, UK, September 2017, doi: 10.23919/IConAC.2017.8082028.

[6] H. Mohamed, P. Lazaridis, D. Upton, U. Khan, B. Saeed, A. Jaber, I. Glover and M. Viera, "Partial Discharge Detection Using Low Cost RTL-SDR Model for Wideband Spectrum Sensing," in International Conference on Telecommunication, Thessaloniki, Greece, 2016.

[7] M. X. Zhu, Y. B. Wang, Q. Liu, J. N. Zhang, J. B. Deng, G. J. Zhang, X. J. Shao and W. L. He, "Localization of Multiple Partial Discharge Sources in Air-Insulated Substation Using Probability-Based Algorithm", IEEE Transactions on Dielectrics and Electrical Insulation, 24, 1, pp. 157-166, February 2017, doi: 10.1109/TDEI.2016.005964.

[8] P. C. Baker, B. Stephen, M. D. Judd and S. D. J. McArthur, "Development of an Integrated LowPower RF Partial Discharge Detector", IEEE Electrical Insulation Conference (EIC), pp. 273-277, Montreal, Canada, June 2009, doi: $\underline{10.1109 / \text { EIC.2009.5166356. }}$.

[9] A. A. Jaber, P. I. Lazaridis, M. Moradzadeh, I. A. Glover, Z. D. Zaharis, M. F. Q. Vieira, M. D. Judd and R. C. Atkinson, "Calibration of Free-Space Radiometric Partial Discharge Measurements", IEEE Transactions on Dielectrics and Electrical Insulation, 24, 5, October 2017, pp. 3004-3014, doi: $\underline{10.1109 / T D E I .2017 .006730}$

[10] I. E. Portugues, P. J. Moore, I. A. Glover, C. Johnstone, R. H. McKosky, M. B. Goof and L. van der Zel, "RF-Based Partial Discharge Early Warning System for Air-Insulated Substations", IEEE Transactions on Power Delivery, 24, 1, January 2009, pp. 20-29, doi: 10.1109/TPWRD.2008.2005464.

[11] G. Robles, J. M. Fresno, M. Sánchez-Fernández and J. Martínez-Tarifa, "Antenna Deployment for the Localization of Partial Discharge in Open-Air Substations", Sensors, 16, 4, pp. 9882-9898, doi: 10.3390/s16040541.
[12] M. X. Zhu, Y. B. Wang, Y. Li, H. B. Deng, X. J. Shao and G. J. Zhang, "Detection and Localization of Partial Discharge in Air-Insulated Substations using UHF Antenna Array", $3^{\text {rd }}$ IEEE Conference on Power Engineering and Renewable Energy (ICPERE), pp. 221-224, Yogyakarta, Indonesia, November 2016, doi: $\underline{\text { 10.1109/ICPERE.2016.7904875 }}$

[13] H. C. So and L. Lin, "Linear Least Squares Approach for Accurate Received Signal Strength Based Source Localization," IEEE transactions on signal processing, vol. 59, no. 8, pp. 4035-4040, 2011.

[14] Y. Xu, J. Zhou and P. Zhang, "RSS-based source localization when path-loss parameters are unknown," IEEE communication letters, vol. 18, no. 6, pp. 1055-1058, 2014. 